reported in 10 children studied at the University of Arkansas, Little Rock. (Davis P, Landers A, Gentry B et al. Perceptual and Motor Skills Dec 1997;85:809-810).

\title{
MOYAMOYA SYNDROME AND CONGENITAL HEART DISEASE
}

The association of moyamoya syndrome and congenital heart disease is described in 5 patients at Children's Hospital, Boston, MA. Stroke was the presenting sign in 3 and seizures in 2. Coarctation of the aorta with septal or valvular defects was diagnosed in 3, and tetralogy of Fallot in 2. Moyamoya, diagnosed after surgery for congenital heart disease, was treated by cerebral revascularization. (Lutterman J, Scott M, Nass R, Geva T. Moyamoya syndrome associated with congenital heart disease. Pediatrics Jan 1998;101:57-60). (Respond: Tal Geva MD, Department of Cardiology, Children's Hospital, 300 Longwood Ave, Boston, MA 02115).

COMMENT. Moyamoya syndrome should be considered in the differential diagnosis of stroke or seizures associated with congenital heart disease, both before or after surgery.

\section{METABOLIC DISORDERS}

\section{BIOTINIDASE DEFICIENCY: EARLY PRESENTATION}

Two infants with manifestations of biotinidase deficiency presenting at age 3 weeks and 2 weeks are reported from the University of Aarhus, Roskilde County Hospital, and Herning Central Hospital, Denmark. Patient 1, born to related Kurdic parents, developed a generalized skin rash at 3 weeks, generalized tonic-clonic seizures up to 20 times daily at 6 weeks, and visual inattention, hypertonia and hyperreflexia on admission at 8 weeks. EEG showed epileptiform activity. Valproic acid was ineffective. Metabolic screening showed urinary B-hydroxyisovalerate and B-methylcrotonylglycine, and very low serum biotinidase activity. After oral biotin ( $5 \mathrm{mg} \mathrm{x} 3$ daily) the seizures stopped within a few days, and at 2 year follow up psychomotor development was normal except for hearing loss. MRI showing cerebral atrophy initially was normal at 12 months. Patient 2, the second child of related Kurdic parents, presented at 1 hour after birth with respiratory distress and septicemia. She had dry and squamous skin at 2 weeks, loss of hair at 4 weeks, and on readmission at 6 weeks she was lethargic, hypotonic, and hypothermic. Visual inattention, trembling, tense fontanelle, tonic clonic seizures, conjunctivitis, and alopecia were noted. EEG showed diffuse slowing and a right occipital spike focus. CT was suggestive of periventricular leukodystrophy. Serum lactate and pyruvate were elevated. Organic aciduria and absent serum biotinidase confirmed the diagnosis of biotinidase deficiency. Oral biotin (10mg daily) was begun at 7 weeks, and seizures were controlled and other manifestations improved within 2 weeks. At 18 month follow-up, development and CT were normal. The authors advocate routine neonatal screening for biotinidase deficiency in Denmark. (Haagerup A, Andersen JB, Blichfeldt S, Christensen MF. Biotinidase deficiency: two cases of very early presentation. Dev Med Child Neurol Dec 1997;39:832-835). (Respond: Dr Annette Haagerup, Institute of Human Genetics, University of Aaarhus, DK-8000 Aarhus C, Denmark).

COMMENT. Biotinidase deficiency is an autosomal recessive disorder causing multiple carboxylase deficiency and usually manifested at 3 to 6 months of age with intractable seizures, hypotonia, skin rash, alopecia, and developmental delay. Lactic acidosis leads to coma and death in untreated cases. 